\title{
Different repartition of the cryptic species of black aspergilli according to the anatomical sites in human infections, in a French University hospital
}

\author{
Maud Gits-Muselli (1D 1,2,3, , Samia Hamane ${ }^{1}$, Benjamin Verillaud ${ }^{3,4,5}$, \\ Elisa Cherpin ${ }^{1}$, Blandine Denis ${ }^{6}$, Louise Bondeelle ${ }^{3,7}$, Sophie Touratier ${ }^{8}$, \\ Alexandre Alanio (D) 1,2,3 , Dea Garcia-Hermoso ${ }^{1}$ and Stéphane Bretagne (D) 1,2,3
}

\begin{abstract}
'Laboratoire de Parasitologie-Mycologie, Hôpital Lariboisière Saint-Louis Fernand Widal, Assistance PubliqueHôpitaux de Paris (AP-HP), France, ${ }^{2}$ Institut Pasteur, CNRS, Unité de Mycologie Moléculaire, Centre National de Référence Mycologie et Antifongiques, UMR2000, France, ${ }^{3}$ Université de Paris, France, ${ }^{4}$ Département d'Otorhinolaryngologie, Hôpital Lariboisière Saint-Louis Fernand Widal, Assistance Publique-Hôpitaux de Paris (AP-HP), France, ${ }^{5}$ Institut National de la Santé et de la Recherche Médicale U1141, France, ${ }^{6}$ Département de Maladies infectieuses, Hôpital Lariboisière Saint-Louis Fernand Widal, Assistance Publique-Hôpitaux de Paris (AP-HP), France, ${ }^{7}$ Pneumologie, Hôpital Lariboisière Saint-Louis Fernand Widal, Assistance Publique-Hôpitaux de Paris (APHP), France and ${ }^{8}$ Pharmacie, Hôpital Saint-Louis, Assistance Publique-Hôpitaux de Paris (AP-HP), France
\end{abstract}

*To whom correspondence should be addressed. E-mail: maud.gits-muselli@aphp.fr

Received 11 September 2020; Revised 25 January 2021; Accepted 27 April 2021; Editorial Decision 2 February 2021

\begin{abstract}
Black aspergilli of the section Nigri are rarely differentiated at the species level when originating from human specimens. We wondered whether some cryptic species could be more frequently observed in some clinical entities. We analyzed the 198 black isolates consecutively collected from the external ear canal $(E E C ; n=66)$, respiratory specimens $(n=99)$, and environment $(n=33)$. DNA was extracted and species identification was performed upon the partial calmodulin gene. We identified by decreasing frequency: Aspergillus welwitschiae (35.3\%), Aspergillus tubingensis (34.3\%), Aspergillus niger (17.2\%), Aspergillus luchuensis (4\%), Aspergillus aff. welwitschiae (3\%), Aspergillus neoniger (2\%), Aspergillus piperis (1.5\%), Aspergillus japonicus $(1.0 \%)$, Aspergillus vadensis (0.5\%), and two Aspergillus tubingensis clade (1\%). The distribution of the three main cryptic species was different between EEC and respiratory samples $(P<0.001)$ but not different between respiratory and environment samples $(P=0.264)$. Aspergillus welwitschiae was more often associated with EEC (54.5\%), whereas $A$. tubingensis and $A$. niger were predominant in respiratory samples (39.4 and $26.3 \%$, respectively). Among the 99 respiratory isolates, only 10 were deemed responsible for probable invasive aspergillosis, of which six were mixed with other pathogenic moulds. This study shows the interest to pursue the identification of clinical isolates in the Aspergillus section Nigri to unravel some specific associations with clinical entities. The association of $A$. welwitschiae with otomycosis suggests a better fitness to infect/colonize the ear canal. Also, members of the Aspergillus section Nigri alone are rarely responsible for invasive aspergillosis.
\end{abstract}

\section{Lay summary}

We analyzed 198 black aspergilli isolates collected from different samples type to determine their species identification. We observe a different distribution of species between ear canal and respiratory samples $(P<0.001)$, suggesting a better fitness of $A$. welwitschiae to infect the ear canal.

Key words: Black aspergilli, Aspergillus welwitschiae, Aspergillus tubingensis, Aspergillus niger, otomycosis, invasive aspergillosis. 


\section{Introduction}

Black aspergilli of the section Nigri are well known in biotechnology ${ }^{1}$ and for involvement in plant diseases ${ }^{2}$, but are rarely reported in human invasive fungal infections. Indeed, the section Nigri is by far outnumbered by Aspergillus fumigatus in invasive pulmonary aspergillosis. ${ }^{3,4}$ When isolated in medical laboratories from non-sterile sites, such as respiratory specimens, the black aspergilli are often disregarded as a contaminant. ${ }^{5,6}$ One notable exception is the external ear fungal infection, where black aspergilli are frequently reported in different geographical areas, such as Germany ${ }^{7}$, Hungary $^{8}$, Turkey $^{9}, \operatorname{Iran}^{10,11}$, Egypt $^{12}$, Nigeria ${ }^{13}$, India ${ }^{14}$, Japan ${ }^{15}$, or China. ${ }^{16}$ In both respiratory and external ear canal (EEC) specimens, the black aspergilli are rarely identified at the species level since no association with a specific clinical entity has been reported, in contrast with agriculture where the identification to the cryptic species is essential for deciphering between the different plant diseases, considering species-specific mycotoxin production. ${ }^{2,17}$

Aspergillus section Nigri is indeed a taxonomical group containing several phenotypically close species hardly differentiated using standard laboratory procedures. The taxonomy of the black aspergilli has recently been rearranged into six different series ${ }^{18}$ and 32 distinct current taxa. ${ }^{19,20}$ The assignation of black aspergilli to the species level is now possible by sequencing partial regions of the beta-tubulin or the calmodulin genes. ${ }^{18,21,22}$ This later locus is recommended considering the possibility of tubulin paralogs within the Nigri section ${ }^{18}$ and has shown higher average bootstrap support compared to the beta-tubulin phylogeny. ${ }^{21}$ We were interested in identifying cryptic species among the isolates recovered from clinical and environmental specimens in our laboratory and in determining whether given species correlated with specific clinical entities.

\section{Patients and methods}

\section{Collection of isolates and antifungal susceptibility testing}

We retrospectively studied all the isolates consecutively recovered from EEC and respiratory specimens between October 2010 and December 2018 in our laboratory. Saint-Louis Hospital is a 650-bed university hospital with major clinical activities in hematology and associated with Lariboisière Hospital, which is a reference center for otorhinolaryngology. The EEC isolates were obtained from patients with external otomycosis and the respiratory isolates from patients with various backgrounds investigated for pulmonary symptoms. The patients were classified according to the revised European Organization for Research and Treatment of Cancer and the Mycoses Study Group (EORTC/MSG) definition ${ }^{23}$ by a multidisciplinary hospital group as already described. ${ }^{24}$ Information regarding previous therapies including topical treatment for patients with otomycosis was not available. We added isolates collected from systematic air sampling as a survey in hematology wards of our hospital.

The clinical samples were cultured on Sabouraudchloramphenicol-gentamicin agar medium (Bio-Rad, Marnesla-Coquette, France) and incubated at $30^{\circ} \mathrm{C}$ for a maximum of 3 weeks. Air samples were cultured on MALT extract agar plates (VWR, Fontenay-sous-Bois, France) and incubated at $30^{\circ} \mathrm{C}$ for a maximum of 10 days. Isolates were phenotypically identified as Aspergillus section Nigri based on macroscopic and microscopic criteria. For each positive culture, spores from several colonies were stored at $-20^{\circ} \mathrm{C}$ using specific cryotubes (PRO-LAB Diagnostics, Richmond Hill, Canada).

The susceptibility profile was determined only when medically indicated, i.e. for the isolates deemed responsible for invasive aspergillosis (IA) or for those serially recovered from patients with otomycosis with reoccurrence of the same symptoms.

Antifungal susceptibility testing was performed according to the European Committee on Antimicrobial Susceptibility Testing (EUCAST) broth microdilution method following the procedure E. DEF 7.3.2 (https://www.eucast.org/ fileadmin/src/media/PDFs/EUCAST_files/AFST/Files/EUCAST_

E_Def_9.3.2_Mould_testing_definitive_revised_2020.pdf) with some modifications. ${ }^{25}$ All drugs were purchased from Alsachim (Strasbourg, France). ${ }^{26}$

\section{Molecular investigations}

After thawing, five to ten cryopreserved beads were seeded on a Sabouraud-chloramphenicol-gentamicin agar plate. DNA was extracted from approximately $1 \mathrm{~cm}^{2}$ of mycelium from a 5day-old culture at $30^{\circ} \mathrm{C}$. The mycelium was suspended in $1 \mathrm{ml}$ of ATL lysing solution (Qiagen, Germantown, MD, USA) in a tube containing $500 \mu \mathrm{l}$ of ceramic beads (MAGNA Lyser, Roche Diagnostics, Mannheim, Germany), and disrupted using a Precellys apparatus (Bertin technologies, Montigny-le-Bretonneux France). Then, $400 \mu \mathrm{l}$ of the supernatant was used to purify DNA using the MagNA Pure LC DNA Isolation Kit-Large Volume (Roche Diagnostics) with a MagNaPure apparatus according to the manufacturer's instructions. The extracted DNA was stored at $-20^{\circ} \mathrm{C}$ until use. The calmodulin gene was amplified using the primers $\mathrm{cmd} 5$ ( $5^{\prime}$-CCGAGTACAAGGAGGCCTTC- $\left.3^{\prime}\right)$ and $\mathrm{cmd} 6\left(5^{\prime} \text {-CCGATAGAGGTCATAACGTGG-3' }{ }^{\prime}\right)^{27}$ and the sequencing performed as already published. ${ }^{28}$

Strain identification was achieved by sequence similarity search (BLASTn) against curated fungal reference databases available at the online MycoBank database (http://www. mycobank.org/). Multiple sequence alignments were carried out using the MAFFT algorithm with default settings in Geneious v. R9 software (Auckland, New Zealand). After alignment, sequences were trimmed to perform phylogenetic tree construction on the size of a standardized sequence ( $457 \mathrm{bp}$ ). 
Table 1. Repartition of the different species identified among 198 isolates according to sampling origin.

\begin{tabular}{|c|c|c|c|c|}
\hline Total number of isolates $\mathrm{n}=198$ & $\begin{array}{l}\text { External otitis } \\
\text { isolates } n=66\end{array}$ & $\begin{array}{c}\text { Respiratory } \\
\text { isolates } n=99\end{array}$ & $\begin{array}{l}\text { Environmental } \\
\text { isolates } \mathrm{n}=33\end{array}$ & $P$ values \\
\hline Aspergillus welwitschiae $(70 ; 35.3 \%)$, & $36 / 66(54.5 \%)$ & $25 / 99(25.2 \%)$ & $9 / 33(27.3 \%)$ & $\begin{array}{c}P<10^{-3} \\
P=0.0005\end{array}$ \\
\hline Aspergillus tubingensis $(68 ; 34.3 \%)$ & $18 / 66(27.3 \%)$ & $39 / 99(39.4 \%)$ & $11 / 33(33.3 \%)$ & $\begin{array}{c}P<10^{-3} \\
P=0.0005\end{array}$ \\
\hline Aspergillus niger (34; 17.2\%), & $5 / 66(7.6 \%)$ & $26 / 99(26.3 \%)$ & $3 / 33(9.1 \%)$ & $\begin{array}{c}P<10^{-3} \\
P=0.0005\end{array}$ \\
\hline Aspergillus luchuensis (8; 4\%), & $1 / 66$ & $1 / 99$ & $6 / 33$ & \\
\hline Aspergillus aff welwitschiae $(6 ; 3 \%)$ & $3 / 66$ & 2/99 & $1 / 33$ & \\
\hline Aspergillus neoniger $(4 ; 2 \%)$ & $2 / 66$ & $1 / 99$ & $1 / 33$ & \\
\hline Aspergillus piperis $(3 ; 1.5 \%)$ & $1 / 66$ & $1 / 99$ & $1 / 33$ & \\
\hline Aspergillus japonicus $(2 ; 1.0 \%)$, & $0 / 66$ & $2 / 99$ & $0 / 33$ & \\
\hline Aspergillus vadensis $(1 ; 0.5 \%)$ & $0 / 66$ & $1 / 99$ & $0 / 33$ & \\
\hline $\begin{array}{l}\text { Aspergillus spp. Section Nigri } \\
\text { tubingensis clade }(2,1 \%)\end{array}$ & $0 / 66$ & $1 / 99$ & $1 / 33$ & \\
\hline
\end{tabular}

Phylogenetic analysis was performed by maximum-likelihood with MEGA10.X. ${ }^{29}$ The best substitution model, according to the Bayesian information criterion, was the Kimura- 2 parameter with gamma-distributed evolutionary rates. Support for internal branches was assessed with 500 bootstrap replicates. A branch present in greater than $70 \%$ of bootstrap replicate is deemed robust ${ }^{30} \mathrm{~A}$ graphic representation of the phylogenetic tree was created using the Interactive Tree Of Life (iTOL) webserver (https://itol.embl.de/). ${ }^{31}$ In addition to the clinical isolates, calmodulin gene sequences corresponding to Aspergillus section Nigri type strains were downloaded from GenBank and incorporated into the phylogenetic analysis (Supplementary Table 1).

\section{Ethical consideration}

The present study is a non-interventional study without any change in the usual diagnostic procedures. The analysis relied on existing data from previously performed tests according to physicians' prescriptions. According to the French Health Public Law (CSP Art L1121-1.1), such protocols do not require approval by an ethics committee and are exempt from the otherwise mandatory informed consent requirements.

\section{Results}

\section{Identification and distribution of cryptic species of Aspergillus section Nigri}

A total of 207 isolates were sequenced: 73 from 66 patients with external otitis samples; 101 from 99 patients investigated for respiratory symptoms; and from 33 isolates from the indoorhospital environment. For patients with serial samples, only the first one was considered. Thus, 198 isolates (66 EEC isolates, 99 respiratory isolates, and 33 air-sampled isolates) were considered for the comparison of species distribution according to the sample site (Table 1).

Based on the BLAST analysis of the calmodulin sequences, we identified a total of nine species. The percentage of similarity to assign the sequence to a reference name was $\geq 99.1 \%$ for all but for two sequences, one with $98.8 \%$ similarity with Aspergillus piperis CBS 112811, and one with $96.7 \%$ similarity with Aspergillus vadensis CBS 113365, both placed in the tubingensisclade. Three species, Aspergillus welwitschiae (70/198; 35.3\%), Aspergillus tubingensis $(68 / 198 ; 34.3 \%)$, and Aspergillus niger $(34 / 198 ; 17.2 \%)$ accounted for $87 \%(\mathrm{n}=172)$ of the total (Table 1).

The phylogenetic tree inferred from the calmodulin gene sequences is presented in Figure 1. The maximum likelihood analyses distinguished three clades: the japonicus-clade (two isolates), the niger-clade (110 isolates), and the tubingensis-clade (86 isolates). These three clades were well supported with bootstrap values of 100,99 , and 96, respectively. The niger-clade comprised three species grouped in A. welwitschiae $(\mathrm{n}=70)$ (bootstrap value $=87)$, A. niger $(\mathrm{n}=34)($ bootstrap value $=99)$, and $A$. aff. welwitschiae $(\mathrm{n}=6)$ (bootstrap value $=85)$. The tubingensisclade comprised five species: A. tubingensis (68 isolates) grouped together with a low support (bootstrap value $=67$ ), and within the second branch, clinical strains grouped together according to the identified species: A. luchuensis (eight isolates) (bootstrap value $=84)$, A. neoniger (four isolates) (bootstrap value $=90$ ), A. piperis three isolates) (bootstrap value $=74$ ), and A. vadensis (one isolate) (bootstrap value $=90$ ).

When considering the two main clades tubingensis and niger, there was no difference in the repartition between EEC and respiratory isolates $(P=0.12)$ or between respiratory and environment isolates $(P=0.13)$. However, at the species level, when 


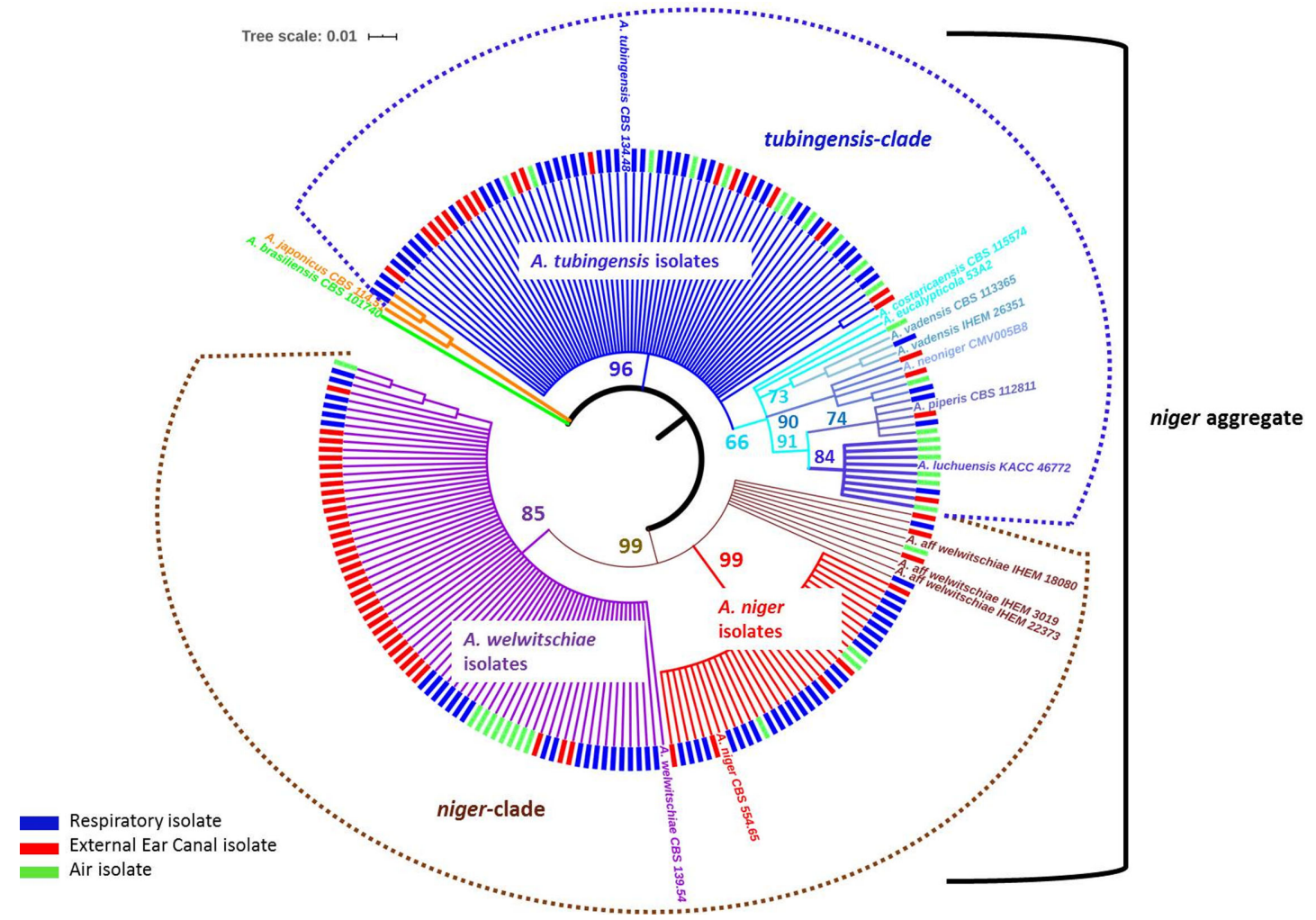

Figure 1. Maximum likelihood tree based on partial calmodulin sequences for 198 strains of Aspergillus section Nigri according to their anatomical site of isolation. Leaf labels highlighted in blue represent respiratory samples, leaf labels highlighted in red external ear canal samples, and leaf labels highlighted in green air samples. Dark blue branches correspond to $A$. tubingensis isolates, red branches to $A$. niger isolates, purple branches to $A$. welwitschiae isolates, and orange branches to $A$. japonicus isolates. Bootstrap values (in percentage) are presented closed to their dedicated branches.

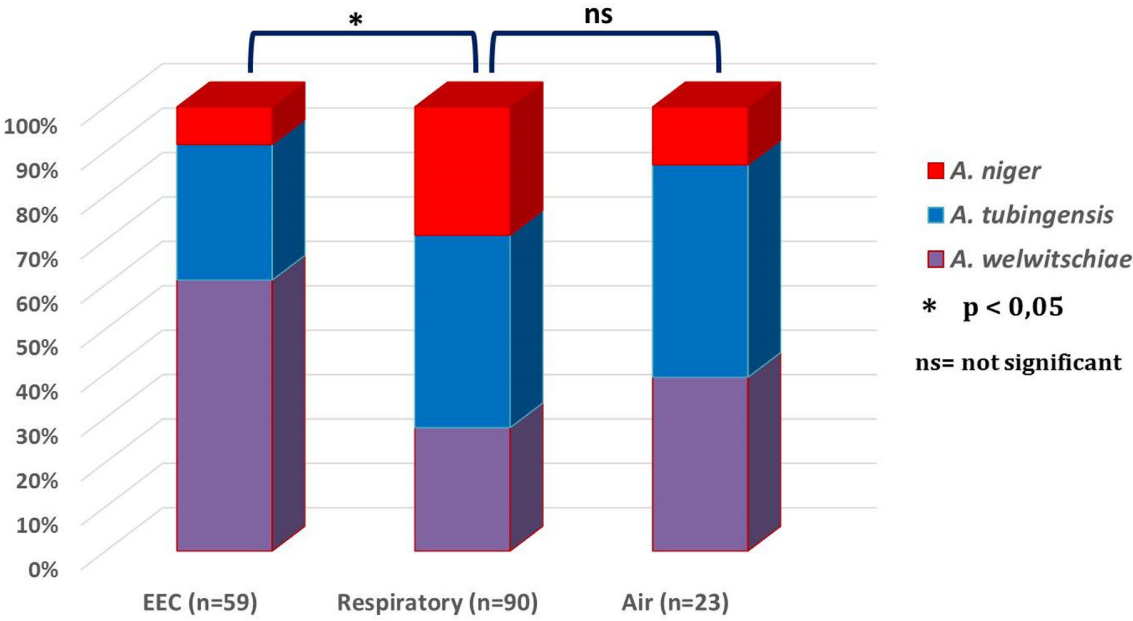

Figure 2. Repartition of the three main species (Aspergillus welwitschiae, Aspergillus tubingensis, and Aspergillus niger) according to the site of sampling.

considering only the three main species, A. welwitschiae, A. tubingensis, and $A$. niger, the repartition was significantly different between EEC and respiratory samples (Pearson's Chi-squared test; $P<0.001$ ), but not between respiratory and air samples $(P=0.264)$. The three main species repartition according to the site of sampling is presented in Figure 2. Twenty-six isolates $(13 \%)$ belonging to less frequent or less well-defined species were sampled from air $(\mathrm{n}=10 / 26,38.5 \%)$, respiratory tract $(\mathrm{n}=9 / 26$, $34.6 \%)$, and EEC $(7 / 26 ; 26.9 \%)$. Of note, six of the eight Aspergillus luchuensis isolates were from air samples.

The calmodulin sequences of 196 black aspergilli isolates from this study, with a defined species, were deposited in GenBank and assigned to the accession numbers MW340535-MW340610 for the A. welwitschiae, MW340611- 
MW340678 for A. tubingensis, MW340679-MW340712 for A. niger, MW340713-MW340720 for A. luchuensis, MW345843 and - MW345846 for A. japonicus, MW345839, MW345840, MW345842 and MW345847 for A. neoniger, MW345841, MW345844 and MW345848 for A. piperis, and MW345845 for $A$. vadensis. GenBank accession numbers assigned to the two black aspergilli isolates without a defined species, belonging to the tubingensis-clade were MW509615 and MW509616.

\section{Clinical data}

The 66 patients (31 men, 35 women, mean age $44.4 \pm 15.7$ years) with external otomycosis had A. welwitschiae identified, i.e., 54.5\% of EEC samples. Four patients (two men and two women) had recurrent infections and were sampled two to four times from 1 month to 3 years apart. These seven recurrent isolates were all identified as A. welwitschiae as the initial isolate with no change in antifungal susceptibility pattern (not shown).

Ninety-nine respiratory specimens from as many patients (50 males, 49 females, mean age $60.4 \pm 14.4$ years) consisted in broncho-alveolar lavage fluids $(\mathrm{n}=8)$, bronchial aspiration ( $\mathrm{n}=51)$, and sputum $(\mathrm{n}=40)$. Underlying disease conditions included 44 chronic pulmonary diseases (12 chronic obstructive pulmonary diseases, five asthma, six fibrosis, eight inflammatory diseases, nine others, and four not specified), 30 hematological malignancies, four common variable immunodeficiency, 16 solid tumors, and five other diseases. There was no significant difference of the cryptic species distribution among the respiratory specimens according to underlying disease and the type of specimens (not shown). Among the 30 patients with hematological malignancies, ten were classified as having probable IA according to the revised EORTC/MSG consensus definitions (Table 2).

Among the ten patients with probable IA, A. welwitschiae $(\mathrm{n}=3)$, A. tubingensis $(\mathrm{n}=5)$, and A. niger $(\mathrm{n}=2)$ were identified (Table 2). Six patients had other molds in the same or concomitant respiratory specimens (Aspergillus hiratsukae and Rhizomucor miehei in one patient each, A. fumigatus in four patients). In four cases (three A. tubingensis and one A. welwitschiae), the black Aspergillus was the only species cultured. Of note, three of the four patients were alive at 3 months after diagnosis. The ten isolates responsible for probable IA had MICs to antifungals in the range of what was observed in the French National Reference Centre for Invasive Mycoses and Antifungals with no significant difference between the cryptic species (Supplementary Table 2).

Among the 89 patients considered as colonized, two had a second isolation of black aspergilli on a distant sample. For one patient, the first sampling yielded A. welwitschiae and the second 2 years later, A. tubingensis. For the second patient, respiratory specimens yielded A. tubingensis and A. niger 5 months later.

\section{Discussion}

To our knowledge, the present study is the first one analyzing a comprehensive collection of black aspergilli over a given period of time in a single university hospital without selection of a particular site of isolation.

Accurate identification of black Aspergilli isolates remains challenging, and we faced uncertainty for 8 strains over the 198 . Nevertheless, recent studies in the field, allowed us to compare our strains with new identified species. Our six strains denominated as Aspergillus aff welwitschiae, according to the terminology proposed by D'hooge et al in 2019,32 presented 98-99.4\% identity with the deposited calmodulin sequence (Genbank accession MN583580) of the new species, Aspergillus vinaceus sp. nov. recently described. ${ }^{33}$

We mainly identified three cryptic species (A. niger, A. tubingensis, and A. welwitschiae) among the black aspergilli isolates studied. The main observation was that cryptic species were not distributed evenly between EEC and respiratory specimens. We here show that, inside the niger-clade, the two cryptic species $A$. welwitschiae and A. niger were associated with different clinical features when comparing EEC and respiratory samples. Indeed, A. welwitschiae was recovered in more than half $(54.5 \%)$ of the EEC samples and only a fourth $(25.2 \%)$ of the respiratory specimens, while $A$. niger accounted for $26.3 \%$ of the respiratory isolates compared to only $7.6 \%$ if the EEC isolates.

The higher prevalence of A. welwitschiae in EEC has already been reported in otomycosis cases. ${ }^{8}$ Very recently, a trend was also noticed in a study including five French hospitals having analyzed selected isolates with more $A$. welwitschiae in-ear samples than A. tubingensis. ${ }^{34}$ Having worked with isolates of the same geographical origin could have introduced biases since the environment is the source of the black aspergilli. However, we think on the contrary that such sampling alleviates the potential bias due to differences in the environment. Indeed, if the local environment was the major factor to explain the higher frequency of $A$. welwitschiae in EEC, we would have probably observed the same repartition as the air samples, which was not the case in contrast to respiratory samples. To explain the higher prevalence of A. welwitschiae in EEC, a first hypothesis could be a better adaptation in the external ear environment featured by the presence of cerumen, a complex composite of different fatty molecules. ${ }^{35}$ The differences could also be explained by mycotoxins production, as reported in plant diseases. ${ }^{17}$ Ochratoxin toxicity is mediated through oxidative stress, but nothing is known about trans-cutaneous absorption and local toxicity. ${ }^{36}$ The molecular mechanism of fumonisins toxicity is the inhibition of ceramide synthase resulting in modifications of sphingolipids rheostat, which might be more relevant given the fatty content of the cerumen. ${ }^{37}$ 
Table 2. Demographic, clinical, and mycological data, antifungal treatment, and outcome of the 10 patients with probable invasive aspergillosis due to black aspergilli.

\begin{tabular}{|c|c|c|c|c|c|c|c|c|c|}
\hline $\begin{array}{l}\text { Patient } \\
\text { number } \\
\text { sex/age } \\
\text { (years) }\end{array}$ & $\begin{array}{l}\text { Underlying } \\
\text { conditions }\end{array}$ & $\begin{array}{l}\text { Date of } \\
\text { diagnosis }\end{array}$ & $\begin{array}{r}\text { Respiratory } \\
\text { specimen }\end{array}$ & $\begin{array}{r}\text { Mycologic } \\
\text { findings }\end{array}$ & $\begin{array}{l}\text { Nigri } \\
\text { cryptic } \\
\text { species } \\
\text { cultured }\end{array}$ & $\begin{array}{l}\text { Associated } \\
\text { mold }\end{array}$ & $\begin{array}{l}\text { Previous } \\
\text { antifungal } \\
\text { therapy }\end{array}$ & $\begin{array}{l}\text { Antifungal } \\
\text { treatment }\end{array}$ & Outcome \\
\hline F/17 & AML & $11 / 07 / 2014$ & Sputum & $\begin{array}{c}\text { Negative } \\
\text { DE and } \\
\text { serum } \\
\text { GM }\end{array}$ & A. niger & $\begin{array}{r}\text { Neosartorya } \\
\text { hiratsukae }\end{array}$ & Fluconazole & VRZ & $\begin{array}{r}\text { Alive at } 3 \\
\text { months }\end{array}$ \\
\hline $\mathrm{F} / 75$ & $\begin{array}{c}\text { Lymphoma under } \\
\text { ibrutinib }\end{array}$ & 09/09/15 & $\begin{array}{r}\text { Bronchial } \\
\text { aspirate }\end{array}$ & $\begin{array}{l}\text { Positive } \\
\text { DE and } \\
\text { negative } \\
\text { serum } \\
\text { GM }\end{array}$ & $\begin{array}{l}\text { A. wel- } \\
\text { witschiae }\end{array}$ & A. fumigatus & No & VRZ & $\begin{array}{l}\text { Death at } \\
93 \text { days }\end{array}$ \\
\hline $\mathrm{M} / 71$ & MDS & $17 / 10 / 2016$ & Sputum & $\begin{array}{l}\text { Negative } \\
\text { DE and } \\
\text { serum } \\
\text { GM }\end{array}$ & A. niger & $\begin{array}{l}\text { Rhizomucor } \\
\text { miehei }\end{array}$ & No & LAMB & $\begin{array}{c}\text { Death at } \\
\text { day } 8\end{array}$ \\
\hline $\mathrm{M} / 27$ & $\begin{array}{l}\text { AML, allo-HSCT } \\
\text { (14/09/2017) }\end{array}$ & $2 / 10 / 2017$ & Sputum & $\begin{array}{l}\text { Positive } \\
\text { DE and } \\
\text { negative } \\
\text { serum } \\
\text { GM }\end{array}$ & $\begin{array}{l}\text { A. tubin- } \\
\text { gensis }\end{array}$ & None & No & $\begin{array}{l}\text { LAMB and } \\
\text { VRZ, then } \\
\text { VRZ alone }\end{array}$ & $\begin{array}{r}\text { Alive at } 6 \\
\text { months }\end{array}$ \\
\hline $\mathrm{F} / 78$ & MDS & $19 / 04 / 2017$ & BAL fluid & $\begin{array}{c}\text { Positive } \\
\text { DE } \\
\text { serum } \\
\text { GM and } \\
\text { BAL } \\
\text { GM }\end{array}$ & $\begin{array}{l}\text { A. tubin- } \\
\text { gensis }\end{array}$ & A. fumigatus & No & VRZ & $\begin{array}{r}\text { Alive at } 6 \\
\text { months }\end{array}$ \\
\hline $\mathrm{M} / 68$ & MDS & $22 / 05 / 2014$ & Sputum & $\begin{array}{c}\text { Negative } \\
\text { DE and } \\
\text { serum } \\
\text { GM }\end{array}$ & $\begin{array}{l}\text { A. tubin- } \\
\text { gensis }\end{array}$ & A. fumigatus & VRZ & VRZ & $\begin{array}{r}\text { Alive at } 5 \\
\text { months }\end{array}$ \\
\hline $\mathrm{F} / 71$ & AML & $28 / 07 / 14$ & $\begin{array}{r}\text { Bronchial } \\
\text { aspirate }\end{array}$ & $\begin{array}{l}\text { Negative } \\
\text { DE and } \\
\text { serum } \\
\text { GM }\end{array}$ & $\begin{array}{l}\text { A. tubin- } \\
\text { gensis }\end{array}$ & None & No & VRZ & $\begin{array}{r}\text { Alive at } 3 \\
\text { months }\end{array}$ \\
\hline$F / 39$ & $\begin{array}{c}\text { MDS, Fanconi } \\
\text { anemia, } \\
\text { allo-HSCT } \\
\text { (5/12/2013), } \\
\text { hepatic GVHD } \\
\text { treated with } \\
\text { etanercept }\end{array}$ & $20 / 12 / 13$ & BAL fluid & $\begin{array}{c}\text { Negative } \\
\text { DE and } \\
\text { BAL } \\
\text { GM }\end{array}$ & $\begin{array}{l}\text { A. tubin- } \\
\text { gensis }\end{array}$ & None & Caspofungin & LAMB & $\begin{array}{r}\text { Death at } \\
\text { day } 40\end{array}$ \\
\hline $\mathrm{F} / 66$ & $\begin{array}{c}\text { MDS, allo-HSCT } \\
(10 / 10 / 17)\end{array}$ & $30 / 10 / 18$ & Sputum & $\begin{array}{c}\text { Negative } \\
\text { DE and } \\
\text { serum } \\
\text { GM }\end{array}$ & $\begin{array}{l}\text { A. wel- } \\
\text { witschiae }\end{array}$ & A. fumigatus & VRZ & Isavuconazole & $\begin{array}{r}\text { Alive at } 7 \\
\text { months }\end{array}$ \\
\hline $\mathrm{M} / 65$ & $\begin{array}{l}\text { Lymphoma under } \\
\text { rituximab }\end{array}$ & $26 / 05 / 2017$ & Sputum & $\begin{array}{l}\text { Positive } \\
\text { DE and } \\
\text { negative } \\
\text { serum } \\
\text { GM }\end{array}$ & $\begin{array}{l}\text { A. wel- } \\
\text { witschiae }\end{array}$ & None & No & VRZ & $\begin{array}{r}\text { Alive at } 4 \\
\text { months }\end{array}$ \\
\hline
\end{tabular}

${ }^{a}$ AML, acute myeloid leukaemia; MDS: myelodysplastic syndrome; allo-HSCT: allogeneic hematopoietic stem cell transplantation (date of allo-HSCT); BAL: bronchoalveolar lavage; DE: direct examination, GM: galactomannan antigen; VRZ, voriconazole; PSZ, posaconazole; LAMB: liposomal amphoterin B.

However, although more frequent, A. welwitschiae is not the only cryptic species identified in EEC. Because of the retrospective design of the study, no systematic and homogeneous report of the clinical symptoms was available, and we are thus unable to draw a correlation between a cryptic species and a more specified clinical setting than external otomycosis.

Another possibility to explain the higher prevalence of A.welwitschiae in EEC might be a higher resistance to antifungal drugs of A. welwitschiae compared to other cryptic species, knowing that patients can use over-the-counter topical treatment containing antifungal drugs for long periods of time. Some authors report lower MICs to azole drugs for A. welwitschiae than for $A$. tubingensis or A. niger. ${ }^{34,38,39}$ In contrast, a recent study reports a decreased antifungal susceptibility to azoles in clinical isolates of the tubingensis-clade. ${ }^{32}$ Additionally, when the iterative isolates from a treated patient were tested, we did not observe an increase in MICs to antifungals. Moreover, most of the over-the-counter preparations used contain nystatin, a polyene 
antifungal, and no difference in the MICs to amphotericin B, another polyene, of the cryptic species has been reported. Thus, the hypothesis of a higher resistance of A. welwitschiae to antifungals seems unlikely to explain a higher frequency in EEC.

Another observation is the similarity of repartition of the cryptic species between the respiratory isolates and the air isolates. This questions the causality link of any molds other than A. fumigatus recovered from respiratory specimens in the symptoms observed. ${ }^{40}$ This can simply reflect the inefficiency of the pulmonary tract in eliminating them and not an infectious process. Thus, when black aspergilli were isolated in respiratory specimens in chronic pulmonary conditions, none were considered and treated, in contrast to the experience of Takeda et al. ${ }^{41}$ Moreover, the mold recovery highly depends on the methods used, including the type of specimens, volume seeded, temperature, and length of incubation, which is far from being harmonized/consensual..$^{42}$ Repeating sampling over several days can also increase the probability of isolating additional molds. For instance, two patients in the present study had serial samples with black aspergilli, which were not of the same cryptic species. Therefore, in optimizing the cultural conditions and multiplying the samples, one increases the probability to cultivate environmental molds.

Defining probable IA due to cryptic species of black aspergilli is difficult. The consensus criteria for IA do not distinguish between the Aspergillus species ${ }^{23}$ even in the 2020 updated definitions. ${ }^{43}$ Here, only four patients had a probable IA due to black aspergilli alone according to the EORTC/MSG definitions. Similarly, Vermeulen et al. also reported few IAs with only 16 patients over 7 years. ${ }^{5}$ Of these 16 patients, only six had hematological conditions, three of them had mixed infections with Mucorales or Scedosporium apiospermum and died, while two out the three with pure black aspergilli cultures survived at 6 weeks. ${ }^{5}$ The good prognosis of these two patients is similar to that of the three patients in our series with IA due to black aspergilli alone. When looking at the cryptic species, we observed three A.tubingensis and one A. welwitschiae. By sequencing the beta-tubulin gene, Balajee et al. identified six A. tubingensis and 13 A. niger among the 19 black aspergilli of the TRANSNET collection. ${ }^{4}$ The low frequency of IA where black aspergilli are reported as the sole pathogen involved preclude further analysis on the role of specific cryptic species.

In conclusion, our observation underlined for the first time the higher frequency of the cryptic species A. welwitschiae as responsible for otomycosis in a French area, which should warrant additional research to explain why the EEC is more suitable for this species. However, our results need to be confirmed in other settings. In Korea, when comparing environmental isolates and clinical isolates from patients with hematologic malignancies, $A$. tubingensis was more often isolated from environmental samples and A. welwitschiae from clinical samples, which is in contrast to our findings with respiratory isolates. ${ }^{38}$ Thus, our work under- lines the need for pursuing the identification process of clinical isolates to unravel some particularities of cryptic species of the black aspergilli, which can be currently achieved more rapidly using matrix-assisted laser desorption/ionisation time-of-flight mass spectrometry than using sequencing. ${ }^{32}$

\section{Supplementary material}

Supplementary data are available at MMYCOL online.

\section{Acknowledgements}

The authors thank all the clinicians who cared for the patients and all the laboratory staff and more specifically Cécile Gautier for technical assistance regarding culture of strains and MIC determinations. We are also indebted to Françoise Dromer for her helpful comments to the manuscript.

\section{Declaration of interest}

All authors declare that they have no conflict of interests.

\section{References}

1. Cairns TC, Nai C, Meyer V. How a fungus shapes biotechnology: 100 years of Aspergillus niger research. Fungal Biol Biotechnol. 2018; 5: 13-14.

2. Duarte EAA, Damasceno CL, de Oliveira TAS et al. Putting the mess in order: Aspergillus welwitschiae (and not A. niger) is the etiological agent of sisal bole rot disease in Brazil. Front Microbiol. 2018; 9: 1227.

3. Lortholary O, Gangneux J-P, Sitbon K et al. Epidemiological trends in invasive aspergillosis in France: the SAIF network (2005-2007). Clin Microbiol Infect. 2011; 17: 1882-1889.

4. Balajee SA, Kano R, Baddley JW et al. Molecular identification of Aspergillus species collected for the Transplant-Associated Infection Surveillance Network. J Clin Microbiol. 2009; 47: 3138-3141.

5. Vermeulen E, Maertens J, Meersseman P, Saegeman V, Dupont L, Lagrou K. Invasive Aspergillus niger complex infections in a Belgian tertiary care hospital. Clin Microbiol Infect. 2014; 20: O333-O335.

6. Laurel VL, Meier PA, Astorga A, Dolan D, Brockett R, Rinaldi MG. Pseudoepidemic of Aspergillus niger infections traced to specimen contamination in the microbiology laboratory. J Clin Microbiol. 1999; 37: 1612-1616.

7. Vennewald I, Schönlebe J, Klemm E. Mycological and histological investigations in humans with middle ear infections. Mycoses 2003; 46: 12-18.

8. Szigeti G, Kocsubé S, Dóczi I, Bereczki L, Vágvölgyi C, Varga J. Molecular identification and antifungal susceptibilities of black Aspergillus isolates from otomycosis cases in Hungary. Mycopathologia. 2012; 174: 143-147.

9. Ozcan KM, Ozcan M, Karaarslan A, Karaarslan F. Otomycosis in Turkey: predisposing factors, aetiology and therapy. J Laryngol Otol. 2003; 117: 39-42.

10. Sabz G, Gharaghani M, Mirhendi H et al. Clinical and microbial epidemiology of otomycosis in the city of Yasuj, southwest Iran, revealing Aspergillus tubingensis as the dominant causative agent. J Med Microbiol. 2019; 68: 585-590.

11. Aboutalebian S, Mahmoudi S, Mirhendi H, Okhovat A, Abtahi H, Chabavizadeh J. Molecular epidemiology of otomycosis in Isfahan revealed a large diversity in causative agents. J Med Microbiol. 2019; 68: 918-923.

12. Ali K, Hamed MA, Hassan H, Esmail A, Sheneef A. Identification of fungal pathogens in otomycosis and their drug sensitivity: our experience. Int Arch Otorhinolaryngol. 2018; 22: 400-403.

13. Fasunla J, Ibekwe T, Onakoya P. Otomycosis in western Nigeria. Mycoses. 2008; 51: 67-70.

14. Prasad SC, Kotigadde S, Shekhar M et al. Primary otomycosis in the Indian subcontinent: predisposing factors, microbiology, and classification. Int J Microbiol. 2014; 2014: 636493-636499.

15. Hagiwara S, Tamura T, Satoh K et al. The molecular identification and antifungal susceptibilities of aspergillus species causing otomycosis in Tochigi, Japan. Mycopathologia. 2019; 184: 13-21. 
16. Zhang L, Wang X, Houbraken $\mathrm{J}$ et al. Molecular identification and in vitro antifungal susceptibility of aspergillus isolates recovered from otomycosis patients in Western China. Mycopathologia. 2020; 185: 527-535.

17. Susca A, Proctor RH, Morelli M et al. Variation in fumonisin and ochratoxin production associated with differences in biosynthetic gene content in Aspergillus niger and A. welwitschiae isolates from multiple crop and geographic origins. Front Microbiol. 2016; 7: 1412.

18. Samson RA, Visagie CM, Houbraken J et al. Phylogeny, identification and nomenclature of the genus Aspergillus. Stud Mycol. 2014; 78: 141-173.

19. Houbraken J, Kocsubé S, Visagie CM et al. Classification of Aspergillus, Penicillium, Talaromyces and related genera (Eurotiales): an overview of families, genera, subgenera, sections, series and species. Stud Mycol. 2020; 95: 5-169.

20. Varga J, Frisvad JC, Kocsubé S et al. New and revisited species in Aspergillus section Nigri. Stud Mycol. 2011; 69: 1-17.

21. Howard SJ, Harrison E, Bowyer P, Varga J, Denning DW. Cryptic species and azole resistance in the Aspergillus niger complex. Antimicrob Agents Chemother. 2011; 55: 4802-4809.

22. Iatta R, Nuccio F, Immediato D et al. Species distribution and in vitro azole susceptibility of Aspergillus section Nigri isolates from clinical and environmental settings. J Clin Microbiol. 2016; 54: 2365-2372.

23. De Pauw B, Walsh TJ, Donnelly JP et al. Revised definitions of invasive fungal disease from the European Organization for Research and Treatment of Cancer/Invasive Fungal Infections Cooperative Group and the National Institute of Allergy and Infectious Diseases Mycoses Study Group (EORTC/MSG) Consensus Group. Clin Infect Dis. 2008; 46: 1813-1821.

24. Alanio A, Menotti J, Gits-Muselli M et al. Circulating Aspergillus fumigatus DNA is quantitatively correlated to galactomannan in serum. Front Microbiol. 2017; 8: 2040 .

25. Garcia-Hermoso D, Hoinard D, Gantier J-C, Grenouillet F, Dromer F, Dannaoui E. Molecular and phenotypic evaluation of Lichtheimia corymbifera (formerly Absidia corymbifera) complex isolates associated with human mucormycosis: rehabilitation of L. ramosa. J Clin Microbiol. 2009; 47: 3862-3870.

26. Guégan S, Garcia-Hermoso D, Sitbon K et al. Ten-year experience of cutaneous and/or subcutaneous infections due to coelomycetes in France. Open Forum Infect Dis. 2016; 3: ofw106.

27. Hong S-B, Go S-J, Shin H-D, Frisvad JC, Samson RA. Polyphasic taxonomy of Aspergillus fumigatus and related species. Mycologia 2005; 97: 1316-1329.

28. Dellière S, Healey K, Gits-Muselli M et al. Fluconazole and echinocandin resistance of Candida glabrata correlates better with antifungal drug exposure rather than with MSH2 mutator genotype in a french cohort of patients harboring low rates of resistance. Front Microbiol. 2016; 7: 2038.

29. Kumar S, Stecher G, Li M, Knyaz C, Tamura K. MEGA X: molecular evolutionary genetics analysis across computing platforms. Mol Biol Evol. 2018; 35: 1547 1549.
30. Hillis DM, Bull JJ. 1993. An empirical test of bootstrapping as a method for assessing confidence in phylogenetic analysis. System Biol. 1993; 42: 182-192.

31. Letunic I, Bork P. Interactive Tree Of Life (iTOL) v4: recent updates and new developments. Nucleic Acids Res. 2019; 47: W256-W259.

32. D'hooge E, Becker P, Stubbe D, Normand A-C, Piarroux R, Hendrickx M. Black aspergilli: a remaining challenge in fungal taxonomy? Med Mycol. 2018; 57: 773780 .

33. Silva JJD, Iamanaka BT, Ferranti LS et al. Diversity within Aspergillus niger clade and description of a new species: Aspergillus vinaceus sp. nov. J Fungi (Basel). 2020; 6: 371.

34. Carrara B, Richards R, Imbert S et al. Species distribution and comparison between EUCAST and gradient concentration strips methods for antifungal susceptibility testing of 112 Aspergillus section Nigri isolates. Antimicrob Agents Chemother. 2020; 64: 6203.

35. Yoon YJ, Yoon J, Lee EJ, Kim JS. Substance P and calcitonin gene-related peptide in the glands of external auditory canal skin. Clin Exp Otorhinolaryngol. 2017; 10: 321-324.

36. Tao Y, Xie S, Xu F et al. Ochratoxin A: toxicity, oxidative stress and metabolism. Food Chem Toxicol. 2018; 112: 320-331.

37. Dellafiora L, Galaverna G, Dall'Asta C. Mechanisms of fumonisin B1 toxicity: a computational perspective beyond the ceramide synthases inhibition. Chem Res Toxicol. 2018; 31: 1203-1212.

38. Cho S-Y, Lee D-G, Kim W-B et al. Epidemiology and antifungal susceptibility profile of aspergillus species: comparison between environmental and clinical isolates from patients with hematologic malignancies. J Clin Microbiol. 2019; 57: e1.

39. Hashimoto A, Hagiwara D, Watanabe A et al. Drug sensitivity and resistance mechanism in Aspergillus section Nigri strains from Japan. Antimicrob Agents Chemother. 2017; 61: 1.

40. Garcia-Hermoso D, Alanio A, Cabaret O et al. High diversity of non-sporulating moulds in respiratory specimens of immunocompromised patients: should all the species be reported when diagnosing invasive aspergillosis? Mycoses 2015; 58: 557-564.

41. Takeda K, Suzuki J, Watanabe A et al. Species identification, antifungal susceptibility, and clinical feature association of Aspergillus section Nigri isolates from the lower respiratory tract. Med Mycol 2020; 58: 310-314.

42. Arendrup MC, Bille J, Dannaoui E, Ruhnke M, Heussel C-P, Kibbler C. ECIL3 classical diagnostic procedures for the diagnosis of invasive fungal diseases in patients with leukaemia. Bone Marrow Transplant. 2012; 47: 10301045 .

43. Donnelly JP, Chen SC, Kauffman CA et al. Revision and update of the consensus definitions of invasive fungal disease from the European organization for research and treatment of cancer and the mycoses study group education and research consortium. Clin Infect Dis. 2019; 46: 1813. 